University of Nebraska - Lincoln

DigitalCommons@University of Nebraska - Lincoln

\title{
Electron effective mass and phonon modes in GaAs incorporating boron and indium
}

Tino Hofmann

University of Nebraska - Lincoln, thofmann4@unl.edu

Mathias Schubert

University of Nebraska - Lincoln, mschubert4@unl.edu

G. Leibiger

Freiberger Compound Materials GmbH, Am Junger Löwe Schacht 5, 09599 Freiberg, Germany

V. Gottschalch

Institute of Inorganic Chemistry, Faculty of Chemistry and Mineralogy, Universität Leipzig, Linnéstraße 3, 04103 Leipzig, Germany

Follow this and additional works at: https://digitalcommons.unl.edu/mrsecfacpubs

Part of the Materials Science and Engineering Commons

Hofmann, Tino; Schubert, Mathias; Leibiger, G.; and Gottschalch, V., "Electron effective mass and phonon modes in GaAs incorporating boron and indium" (2007). Faculty Publications: Materials Research Science and Engineering Center. 65.

https://digitalcommons.unl.edu/mrsecfacpubs/65

This Article is brought to you for free and open access by the Materials Research Science and Engineering Center at DigitalCommons@University of Nebraska - Lincoln. It has been accepted for inclusion in Faculty Publications: Materials Research Science and Engineering Center by an authorized administrator of DigitalCommons@University of Nebraska - Lincoln. 


\title{
Electron effective mass and phonon modes in GaAs incorporating boron and indium
}

\author{
T. Hofmann ${ }^{\text {a) }}$ and M. Schubert ${ }^{\text {b) }}$ \\ Department of Electrical Engineering and Nebraska Center for Materials and Nanoscience, \\ University of Nebraska-Lincoln, Nebraska 68588-0511 \\ G. Leibiger \\ Freiberger Compound Materials GmbH, Am Junger Löwe Schacht 5, 09599 Freiberg, Germany \\ V. Gottschalch \\ Institute of Inorganic Chemistry, Faculty of Chemistry and Mineralogy, Universität Leipzig, Linnéstraße 3, \\ 04103 Leipzig, Germany
}

(Received 29 March 2007; accepted 5 April 2007; published online 2 May 2007)

\begin{abstract}
The strain-free boron- and indium-containing GaAs compounds are promising candidates for III-V semiconductor solar cell absorber materials with lattice match to GaAs, for which experimental data of the electronic band structure are widely unknown. For nondegenerate, silicon-doped, $n$-type $\mathrm{B}_{0.03} \mathrm{In}_{0.06} \mathrm{Ga}_{0.91} \mathrm{As}$ with band-gap energy of $1.36 \mathrm{eV}$, determined by near-infrared ellipsometry, a strong increase of the electron effective mass of $44 \%$ in $\mathrm{B}_{0.03} \mathrm{In}_{0.06} \mathrm{Ga}_{0.91} \mathrm{As}$ compared to $\mathrm{In}_{0.06} \mathrm{Ga}_{0.94} \mathrm{As}$ is obtained from far-infrared magneto-optic generalized ellipsometry studies. The authors thereby obtain the vibrational lattice mode behavior. For BAs, an experimentally obscure compound, the curvature of the $\Gamma$-point conduction band thus extrapolates to the free electron mass. (C) 2007 American Institute of Physics. [DOI: 10.1063/1.2735669]
\end{abstract}

Among the important group-III-group-V alloys with lattice match to GaAs, the III-V-boride compounds begin to attract interest. Despite many theoretical and experimental studies on binary boride materials, except for the BAs system for which physical material properties are largely obscure, only little is known for ternary and quaternary boronincorporating III-V solid solutions. Demanding studies of fundamental properties, the incorporation of boron may open pathways for band-gap engineering in III-V alloys. Specifically, long-wavelength materials for solar cell absorber, coherent light emitter, and detector devices may enrich the realm of III-V optoelectronic applications. ${ }^{1,2}$ The $\mathrm{B}_{x} \mathrm{In}_{y} \mathrm{Ga}_{1-x-y}$ As quaternary system is of particular interest for solar energy conversion devices because the incorporation of boron and indium in GaAs could potentially lead to strainfree materials with reduced band-gap values. Such absorber materials could be incorporated into multi-junction solar cell structures with high conversion efficiency. ${ }^{3}$ Alloying of indium with GaAs reduces the band gap considerably, but lattice mismatch excludes applications in solar cell structures, where sufficiently large absorber layers are required. Addition of boron counterbalances strain by virtue of the opponent covalent radii between indium and boron as compared to gallium. Possible difficulties may arise eventually upon the boron-induced increase of the band-gap energy and the miscibility gap of the solid solution. However, only few experimental attempts have been reported so far on this material system. ${ }^{1,4-6}$ Theoretical and experimental data on the electronic band structure properties of $\mathrm{B}_{x} \mathrm{In}_{y} \mathrm{Ga}_{1-x-y}$ As alloys are widely unknown. Likewise, only little is known about the lattice mode properties of $\mathrm{B}_{x} \mathrm{In}_{y} \mathrm{Ga}_{1-x-y} \mathrm{As}$, where the host material InGaAs is renowned for its exotic phonon mode behavior, ${ }^{7}$ which follows neither the one- nor the two-mode

\footnotetext{
${ }^{a}$ Electronic mail: thofmann@engr.unl.edu

b)Electronic mail: schubert@engr.unl.edu
}

scheme. For $\mathrm{B}_{x} \mathrm{Ga}_{1-x} \mathrm{As}$, a two-mode phonon behavior has been found for boron concentrations $\leqslant 3 \%$. Isovalent boron substitution of gallium and indium thus provides expectation of an interesting phonon mode behavior.

In this letter we report on measurement of the Brillouinzone $\Gamma$-point and $\Lambda$ electronic transition energies, phonon and impurity modes, and $\Gamma$-point effective conduction band mass from comprehensive spectroscopic ellipsometry investigations. We employ our recently developed model dielectric function (MDF) approach for the electronic band to band transitions, the far-infrared (FIR) ellipsometry technique for phonon and impurity mode measurement, and our FIR magneto-optic generalized ellipsometry (FIR-MOGE) tool for assessment of the free-charge-carrier properties effective mass $\left(m^{*}\right)$, density $(N)$, and mobility $(\mu)$. The sample investigated here consists of a silicon-doped $\mathrm{B}_{0.03} \mathrm{In}_{0.06} \mathrm{Ga}_{0.91} \mathrm{As}$ layer grown pseudomorphically on undoped GaAs by metal organic vapor phase epitaxy. The growth temperature was $T_{G}=550{ }^{\circ} \mathrm{C}$. Further details on growth parameters and composition determination are given in Ref. 8. The quaternary layer was nominally $1300 \mathrm{~nm}$ thick and the nominal freecharge-carrier concentration was about $9 \times 10^{17} \mathrm{~cm}^{-3}$. High resolution $\mathrm{x}$-ray diffraction measurements ensured the high structural quality and the low lattice mismatch of the sample. The perpendicular lattice mismatch $(\Delta a / a)_{\perp}$ was less than $3 \times 10^{-4}$.

According to the classical Drude theory, the dielectric function of semiconductors provides access to the coupled quantities $N / \mathrm{m}^{*}$ and $N \mu$. Previously, $N$ obtained from electrical Hall-effect measurements was combined with ellipsometry results to obtain $m^{*}$ and $\mu$ for thin layers. ${ }^{9}$ However, electrical contacts potentially affect the free-charge-carrier properties upon surface state formation and Fermi level pinning and complicate Hall-effect interpretation. FIR-MOGE was recently demonstrated as a technique for measurement 


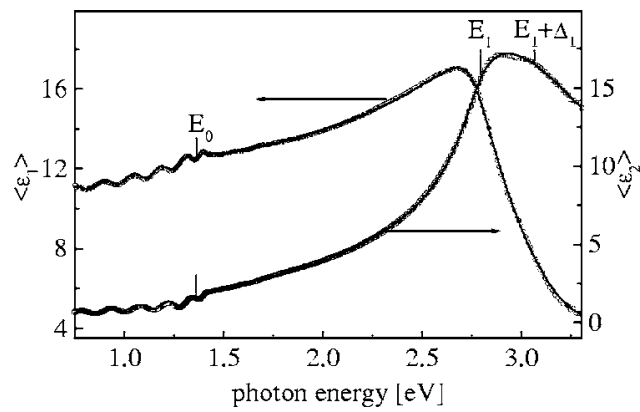

FIG. 1. Experimental (symbols) and best-model calculated NIR-vis ellipsometry data in pseudodielectric function presentation for the BInGaAs samples studied here.

of $m^{*}, N$, and $\mu$ in semiconductor layer structures, dispensing with the need for electrical contacts. ${ }^{10-13}$

Near-infrared visible (NIR-vis) $(0.75-3.3 \mathrm{eV})$ ellipsometry data were measured at room temperature at angles of incidence $\Phi_{a}=65^{\circ}$ and $75^{\circ}$ using a VASETM system (J.A. Woollam Co.) with spectral resolution set to $3 \mathrm{meV}$. FIR (100-600 $\mathrm{cm}^{-1}$ ) ellipsometry measurements were performed with $1 \mathrm{~cm}^{-1}$ resolution at $\Phi_{a}=45^{\circ}$. FIR-MOGE data were acquired within our optical Hall-effect setup with magnetic fields $\mu_{0}|\mathbf{H}|= \pm 3.00(0.02) \mathrm{T}$, as detailed previously. ${ }^{10-12,14}$ The treatment of the optical response of birefringent media is properly described within the Mueller matrix $(\boldsymbol{M})$ and Stokes vector $(\mathbf{S})$ presentation. Ellipsometry permits determination of the full $4 \times 4$ matrix $\boldsymbol{M}$, which relates the four real-valued elements of $\mathbf{S}$ before, $\mathbf{S}^{\text {input }}$, and after, $\mathbf{S}^{\text {output }}$, the interaction with an optical system: $S_{j}^{\text {output }}=\sum_{i=0}^{3} M_{j i} S_{i}^{\text {input }}(j=0, \ldots, 3) .{ }^{15}$ For isotropic media, complex reflectance ratio $\rho=r_{p} / r_{s}$, where $r_{p}$ and $r_{s}$ are the Fresnel coefficients for light polarized parallel $(p)$ and perpendicular $(s)$, respectively, is commonly presented in terms of the pseudodielectric function $\langle\varepsilon\rangle=\left\langle\varepsilon_{1}\right\rangle+i\left\langle\varepsilon_{2}\right\rangle .^{16}$

The ellipsometric data were analyzed using a stratified layer calculation with appropriate MDF accounting for the dielectric response of the epilayer and the substrate. ${ }^{6,13}$ The NIR-vis MDF employs the critical-point model developed recently for BGaAs. ${ }^{6}$ The FIR MDF accounts for polar lattice mode and free-charge-carrier contributions, rendered by harmonic oscillators and the classical Drude approach, respectively. The FIR-MOGE model requires an anisotropic expansion of the plasma contribution according to the classical Drude model, which provides additional access to the carrier cyclotron frequency $\omega_{c}$. During data analysis, significant MDF parameters were varied until calculated and measured data match as close as possible (best fit). A two-phase model (undoped GaAs substrate/ $\mathrm{B}_{0.03} \mathrm{In}_{0.06} \mathrm{Ga}_{0.91} \mathrm{As}$ layer) is employed to analyze the FIR ellipsometry spectra. For the NIRvis data analysis a very thin oxide layer of approximately $3 \mathrm{~nm}$, formed on top of the $\mathrm{B}_{0.03} \mathrm{In}_{0.06} \mathrm{Ga}_{0.91} \mathrm{As}$ layer, was included to properly implement its influence on the NIR-vis data. ${ }^{17}$

Figure 1 presents experimental and best-model calculated NIR-vis ellipsometry spectra in pseudodielectric function representation $\langle\varepsilon\rangle=\left\langle\varepsilon_{1}\right\rangle+i\left\langle\varepsilon_{2}\right\rangle$. The onset of absorption in the $\mathrm{B}_{0.03} \mathrm{In}_{0.06} \mathrm{Ga}_{0.91}$ As layer at $E_{0}=1.360(0.002) \mathrm{eV}$ indicates the $\Gamma$-point band to band transition energy. The thickness of the $\mathrm{B}_{0.03} \mathrm{In}_{0.06} \mathrm{Ga}_{0.91}$ As layer is obtained as $d_{\mathrm{BInGaAs}}=1288(2) \mathrm{nm}$. The $\Lambda$ transition energies $E_{1}$

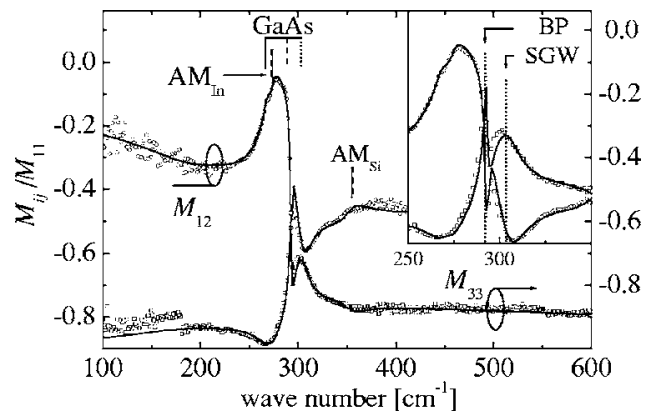

FIG. 2. Same as Fig. 1 for the FIR ellipsometry data in the Mueller matrix descriptor system.

$[2.79(0.01) \mathrm{eV}]$ and $E_{1}+\Delta_{1}[3.07(0.01) \mathrm{eV}]$ are indicated by vertical arrows. These energies are found here to be approximately 70 and $10 \mathrm{meV}$ smaller than those of unstrained $\mathrm{In}_{0.06} \mathrm{Ga}_{0.94} \mathrm{As}$, respectively. ${ }^{18}$

Figure 2 depicts experimental data and best-model calculated FIR ellipsometry spectra in the Mueller matrix descriptor system. The GaAs and $\mathrm{B}_{0.03} \mathrm{In}_{0.06} \mathrm{Ga}_{0.91} \mathrm{As}$ phonon modes are superimposed and cause distinct structures in the polarized sample response for wave numbers from 250 to $320 \mathrm{~cm}^{-1}$. The GaAs-like phonon band of the $\mathrm{B}_{0.03} \mathrm{In}_{0.06} \mathrm{Ga}_{0.91} \mathrm{As}$ extends between the transverse optic (TO) and longitudinal optic (LO) mode frequencies at $267.0(0.5)$ and $289.0(0.2) \mathrm{cm}^{-1}$, respectively. These modes compare excellently with data obtained from $\operatorname{In}_{x} \mathrm{Ga}_{1-x}$ As. ${ }^{7,19}$ The high-frequency edge of the phonon band in Fig. 2 is shifted above the GaAs-like LO mode due to LO-phononplasmon coupling. Two additional small bands are identified near $\omega=272$ and $355 \mathrm{~cm}^{-1}$, denoted by $\mathrm{AM}_{\mathrm{In}}$ $\left[\omega_{\mathrm{TO}}=274.1(0.8) \mathrm{cm}^{-1}, \quad \omega_{\mathrm{LO}}=273.1(1.3) \mathrm{cm}^{-1}\right]$ and $\mathrm{AM}_{\mathrm{Si}}$ $\left[\omega_{\mathrm{TO}}=355.2(2.2) \mathrm{cm}^{-1}, \omega_{\mathrm{LO}}=356.0(2.3) \mathrm{cm}^{-1}\right]$, respectively. Both bands possess a very small width $\left(\sim 1 \mathrm{~cm}^{-1}\right)$. Mode $\mathrm{AM}_{\text {In }}$ is tentatively assigned to the InAs-related sublattice vibration. A similar structure was previously reported without assignment from $\operatorname{In}_{x} \mathrm{Ga}_{1-x} \mathrm{As}$ FIR reflectivity measurements. ${ }^{19}$ We assign $\mathrm{AM}_{\mathrm{Si}}$ with a low-polarity $\mathrm{Si}$ mode, likely caused by $\mathrm{Si}$ substituted on $\mathrm{Ga}$ or As sites. Similar modes were previously found in GaAs for wave numbers between 380 and $400 \mathrm{~cm}^{-1}$. 0

Figure 3 summarizes our FIR-MOGE measurements and the corresponding best-model calculated spectra. Here, data are presented by the differences between ellipsometry spectra taken at $\mu_{0}|\mathbf{H}|=3.00(0.02)$ and $-3.00(0.02) \mathrm{T}$. These are the nonvanishing Mueller matrix element difference spectra under reversal of the external magnetic field and which render the magnetic-field-induced optical free-charge-carrier birefringence at FIR wavelengths. If a semiconductor layer structure sample is exposed to a magnetic field $\mu_{0} \mathbf{H}$, the dielectric function of each constituent (substrate, layers, etc.) which contains free charge carriers requires a complexvalued tensor description $\varepsilon^{\mathrm{FC}-\mathrm{MO}}(\omega)$. Measurement of this tensor by acquiring MOGE spectra and comparing model calculated data allows the determination of the cyclotron frequency $\omega_{c}=\mu_{0}|\mathbf{H}|\left(e / m^{*}\right)$ and the plasmon broadening and plasmon frequency parameters $\gamma_{p}=e /\left(m^{*} \mu\right)$ and $\omega_{p}=\sqrt{N e^{2} /\left(\widetilde{\varepsilon}_{0} m^{*}\right)}$, respectively ( $e$ denotes the the elementary charge and $\widetilde{\varepsilon}_{0}$ the vacuum permittivity). ${ }^{12}$ Both plasmon frequency and broadening parameters are already defined by the analysis of the field-free FIR ellipsometry measurement. 


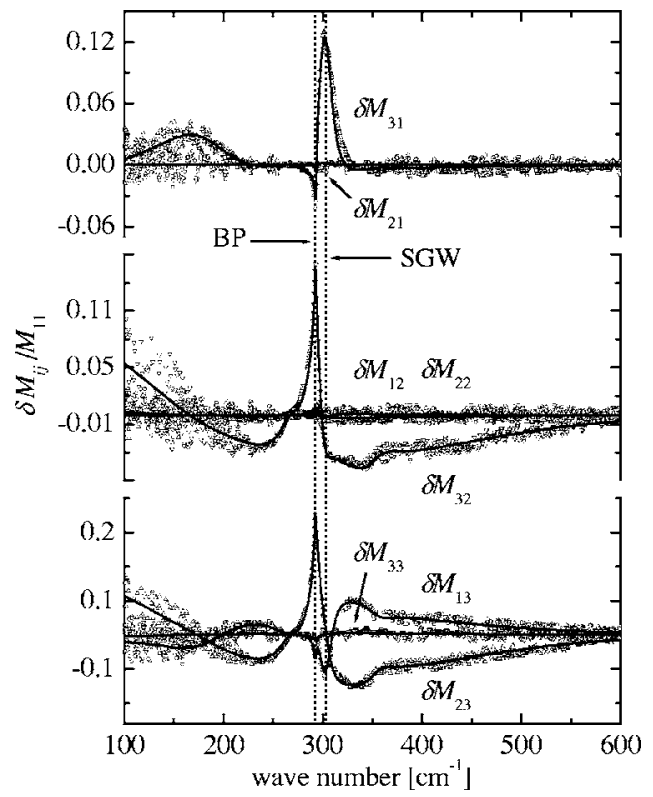

FIG. 3. Same as Fig. 1 for the FIR-MOGE data.

Thus, in order to best match model and experiment in Fig. 3, the same MDF parameter set determined from analysis of the FIR data in Fig. 2 is used, and only variation of $\omega_{c}$ is required. The effective mass parameter follows then directly from $\omega_{c}$. Data in Fig. 2 provided $\omega_{p}=753.5 \mathrm{~cm}^{-1}$ and $\gamma_{p}=113.5 \mathrm{~cm}^{-1}$. Those in Fig. 3 resulted in $\omega_{c}=30.1 \mathrm{~cm}^{-1}$. Hence, for $\mathrm{B}_{0.03} \mathrm{In}_{0.06} \mathrm{Ga}_{0.91} \mathrm{As}$, we obtain $N=5.9(0.3) \times 10^{17} \mathrm{~cm}^{-3}, \quad \mu=885(9) \mathrm{cm}^{2} / \mathrm{V} \mathrm{s}$, and $m^{*}=0.093(0.03) m_{0}$. Mobility and density compare well with our results from electrical Hall-effect measurements $\left(N=8.9 \times 10^{17} \mathrm{~cm}^{-3}, \mu=774 \mathrm{~cm}^{2} / \mathrm{V} \mathrm{s}\right)$. For the Hall-effect results we estimate an uncertainty limit of $15 \%$ obtained from repeated contact processing. The mobility of the quaternary compound is still fairly high.

According to results from plasma edge reflectance measurements on bulklike unstrained InGaAs, the effective mass parameter scales linearly (with slight bowing) with the indium content between those of InAs $\left(0.025 m_{0}\right)$ and GaAs $\left(0.067 m_{0}\right){ }^{21}$ Hence, for $\mathrm{In}_{0.06} \mathrm{Ga}_{0.94} \mathrm{As}$, the effective mass is $m^{*}=0.065 m_{0}$. Upon incorporation of $2.7 \%$ boron, we observe here an increase of the effective mass of about $44 \%$. According to simple kp schemes, $m$ should linearly follow the change of $E_{0}{ }^{22}$ We have previously determined the influence of strain and alloying on $E_{0}$ in BGaAs for boron contents up to $3.3 \% .^{6}$ Assuming deformation potentials of GaAs, the band gap of $\mathrm{B}_{0.03} \mathrm{In}_{0.06} \mathrm{Ga}_{0.91} \mathrm{As}$ is blueshifted by $\sim 3 \%$ with respect to the corresponding strain-free InGaAs compound. Thus, the observed effective mass increase is more than ten times larger compared with the $\sim 3 \%$ increase expected from the simple $\mathbf{k p}$ rule. ${ }^{22}$ Linear extrapola- tion of the effective mass of BAs at the $\Gamma$ point yields $1.02 m_{0}$, approximately the mass of the free electron in vacuum.

In summary, we observe a strong increase of the $\Gamma$-point conduction band effective mass in strain-free Si-doped $\mathrm{B}_{0.03} \mathrm{In}_{0.06} \mathrm{Ga}_{0.91} \mathrm{As}$, with decent electron mobility values and direct band-gap energy $E_{0}=1.360(0.002) \mathrm{eV}$. Linear extrapolation predicts the curvature of this band in BAs to be similar to the free electron mass. Boron- and indium-containing GaAs are promising materials for use in solar cell structures.

We acknowledge support from the Deutsche Forschungsgemeinschaft under grant SCHUH 1338/3-1, the National Science Foundation in MRSEC QSPIN at UNL, startup funds from the CoE at UNL, and the J.A. Woollam Foundation.

${ }^{1}$ G. L. W. Hart and A. Zunger, Phys. Rev. B 62, 13522 (2000).

${ }^{2}$ S. Sakai, Y. Ueta, and Y. Terauchi, Jpn. J. Appl. Phys., Part 1 32, 4413 (1993).

${ }^{3}$ A. Barnett, C. Honsberg, D. Kirkpatrick, S. Kurtz, D. Moore, D. Salzman, R. Schwartz, J. Gray, S. Bowden, K. Goossen, M. Haney, D. Aiken, M. Wanlass, and K. Emery, Conference Record of the 2006 IEEE 4th World Conference on Photovoltaic Energy Conversion (unpublished), Vol. 2, p. 2560.

${ }^{4}$ J. F. Geisz, D. J. Friedman, S. Kurtz, J. M. Olson, A. B. Swartzlander, R. C. Reedy, and A. G. Norman, J. Cryst. Growth 225, 372 (2001).

${ }^{5}$ V. Gottschalch, G. Leibiger, and G. Benndorf, J. Cryst. Growth 248, 468 (2003).

${ }^{6}$ G. Leibiger, V. Gottschalch, V. Riede, M. Schubert, J. N. Hilfiker, and T. E. Tiwald, Phys. Rev. B 67, 195205 (2003).

${ }^{7}$ J. Groenen, R. Carles, G. Landa, C. Guerret-Piécourt, C. Fontaine, and M. Gendry, Phys. Rev. B 58, 10452 (1998).

${ }^{8}$ G. Leibiger, C. Krahmer, J. Bauer, H. Herrnberger, and V. Gottschalch, J. Cryst. Growth 272, 732 (2004).

${ }^{9}$ A. Kasic, M. Schubert, S. Einfeldt, D. Hommel, and T. E. Tiwald, Phys. Rev. B 62, 7365 (2000).

${ }^{10}$ M. Schubert, T. Hofmann, and C. M. Herzinger, J. Opt. Soc. Am. A 20, 347 (2003).

${ }^{11}$ T. Hofmann, M. Schubert, C. M. Herzinger, and I. Pietzonka, Appl. Phys. Lett. 82, 3463 (2003).

${ }^{12}$ T. Hofmann, U. Schade, W. Eberhardt, C. Herzinger, P. Esquinazi, and M. Schubert, Rev. Sci. Instrum. 77, 063902 (2006).

${ }^{13}$ M. Schubert, Infrared Ellipsometry on Semiconductor Layer Structures: Phonons, Plasmons and Polaritons, Springer Tracts in Modern Physics Vol. 209 (Springer, Berlin, 2004), $20 \mathrm{ff}$.

${ }^{14}$ T. Hofmann, U. Schade, K. Agarwal, B. Daniel, C. Klingshirn, M. Hetterich, C. Herzinger, and M. Schubert, Appl. Phys. Lett. 88, 042105 (2006).

${ }^{15}$ C. Chen, I. An, and R. W. Collins, Phys. Rev. Lett. 90, 217402 (2003).

${ }^{16}$ D. E. Aspnes, in Handbook of Optical Constants of Solids, edited by E. Palik (Academic, New York, 1998), Chap. 5, 89 ff.

${ }^{17}$ G. E. Jellison, Thin Solid Films 313-314, 33 (1998).

${ }^{18}$ C. Pickering, R. T. Carline, M. T. Emeny, N. S. Garawal, and L. K. Howard, Appl. Phys. Lett. 60, 2412 (1992).

${ }^{19}$ M. H. Brodsky and G. Lucovsky, Phys. Rev. Lett. 21, 990 (1968).

${ }^{20}$ O. G. Lorimor and W. G. Spitzer, J. Appl. Phys. 37, 3678 (1966).

${ }^{21}$ M. T. Thomas and J. C. Woolley, Can. J. Phys. 49, 2052 (1971).

${ }^{22} \mathrm{P}$. Yu and M. Cardona, Fundamentals of Semiconductors (Springer, Berlin, 1999), $65 \mathrm{ff}$. 